\title{
Determination of the Working Liquids Technical Condition By Analysis of Digital Speckle Images Parameters
}

\section{P. V. Pavlov', A. N. Malov', and A. V. Neupokoeva²}

Military Training and Research Center of the Air Force "Air Force Academy. Professor. N.E. Zhukovskiy and Y.A. Gagarin» (Voronezh), Russia

${ }^{1}$ National research Irkutsk state technical University, Russia

${ }^{2}$ Irkutsk state medical University, Russia

\section{Abstract}

The article discusses the practical realization for the optical control method of technical working fluids condition by the analysis of digital speckle images parameters is discussed. The experimental research results on the application of this method for estimation of aviation fuel and motor oil technical condition are presented.

Corresponding Author:

P. V. Pavlov

pavlov.pave@yandex.ru

Received: 28 January 2018

Accepted: 15 March 2018

Published: 25 April 2018

Publishing services provided by Knowledge $\mathrm{E}$

(c) P. V. Pavlov et al. This article is distributed under the terms of the Creative Commons

Attribution License, which permits unrestricted use and redistribution provided that the original author and source are credited.

Selection and Peer-review under the responsibility of the PhIO Conference Committee.
Keywords: motor oil, aviation fuel, speckle, autocorrelation, impurities, diagnostics

\section{Introduction}

An important role in ensuring the aircraft flights safety in the world in the maintenance of their fuel, hydraulic and oil systems is played. Working liquids (hydraulic oil, motor, and aviation fuel) condition influence on the aircraft serviceability and as a consequence of its trouble-free operation.

Therefore, to ensure the aircraft safety significaly depends on the aviation refilling fuel quality control. World practice shows that currently in the refueling aircraft stages continued application of the optical-visual method for testing of the aviation fuel technical condition for the presence of water and mechanical impurities. Typically, this method is based on visual inspection of the fuel samples, which are take from the tank, and an aeronautical engineer, based on the your vision capabilities and practical experience, makes a decision about the aviation fuel quality. In this "visual" method, there is a error probability due to poor vision, physical fatigue, haste, carelessness controlman. Therefore, it is necessary to develop more accurate with the metrological point of view, the methods and devices of determining water content of aviation fuels in the conditions of the airfield. Well-known works, which proposed to solve this problem with the use of new control methods [1, 2], however, the use of devices to detect 
the impurities presence in working fluids is based on the speckle patterns of optical radiation is not known to us, except for a liquid samples diagnosis in biomedicine $[3,4]$.

On the other hand, the rapid identification and quality control liquids method is knower [5].The its advantage is the low accuracy of determining the impurities presence in the entire test fluid volume due to the controlled fluid structure distortion during the film formation. There is also a method of determining impurities ultra-low concentrations in solutions and gases [6]. This method disadvantage is the dependence of the impurities determination accuracy from the lens resolution and the data recording system. A more modern method of liquid media control is the method of determining the micro particles in liquids by digital holography [7]. In this way the measuring cuvette light laser radiation and receive image of the medium volume in multiple layers. Evaluation of images is performed according to the results of correlation analysis. The main disadvantage of this method is the insufficient accuracy of the impurities determination due to the images shading of successive layers.

\section{Working fluids control method}

We used a new method of determining the technical condition of the fluids, which consists in analyzing of the characteristics of the resulting intensities (speckle images) distributions, recorded by passing a speckle field through the controlled liquid samples. To determine the speckle images parameters (the autocorrelation coefficient, energy spectrum, etc.), was from the liquid sample in the perturbed liquid state of the and in a rest position of it.

The method is realized in the following order. After sampling the liquid sample is stirred, to form a the laser radiation speckle field, record the speckle images of a laser beam passing through the controlled liquid. Then kept for some time to complete the impurities sedimentation in the sample liquid and the speckle-image re-register. The estimation of the impurities parameters is performed according to the correlation coefficient value for the two speckle images, was in an excited sample liquid state and in a rest position.

\section{Installation for realization of the method}

The method was used by experimental setup, the appearance and the scheme is shown in Figure 1. 
As the working fluid used aviation fuel TS-1 and Toyota engine oil 5W40. To obtain the coherent radiation used a single-mode semiconductor laser with diode-pumped SLM-417 with a $532 \mathrm{~nm}$ wavelength and $20 \mathrm{~mW}$ power. As a diffuse object to a speckle field create applied matte glass, for the registration of the last speckle field measured through the cuvette used video camera ELP-USB8MP02G-L75 CCD SONY IMX179 with a resolution of $2048 \times 1536$ pixels (pixel size $1.4 \mu \mathrm{m}$ ).
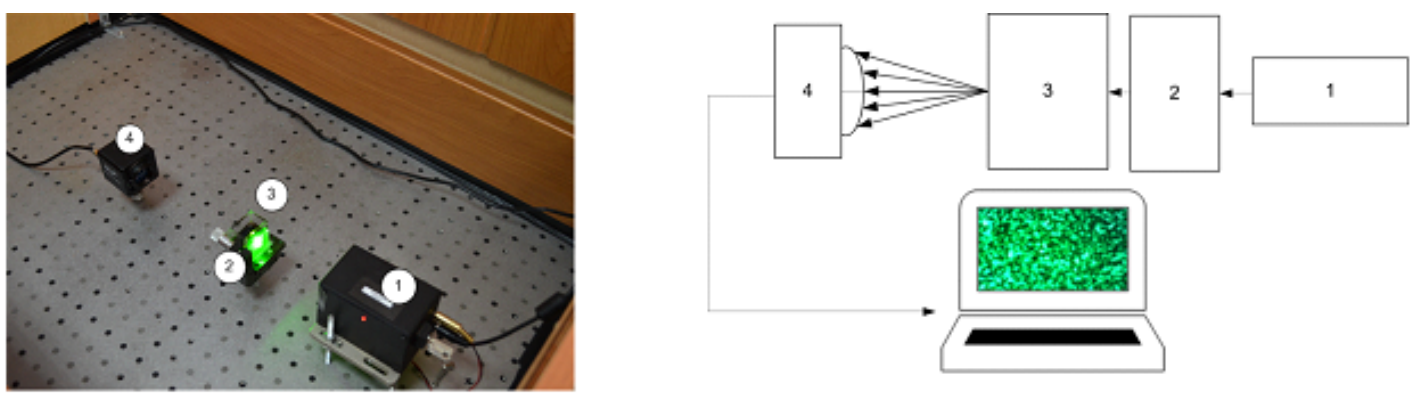

Figure 1: Appearance and schematic diagram of installation. 1 - laser SLM-417, 2 - matte glass, 3 measuring cuvette with liquid sample, 4 - CCD matrix.

\section{Methods of speckle images processing}

The fence was carried out in a cuvette of kerosene with a corresponding water content, stirring it to a homogeneous condition, with further probing the generated speckle field due to coherent radiation diffraction on the matte glass structure, and the frame registration (2 or more frames per second) of the speckle field passing through the cuvette.

Recorded during the experiment the video file was divided into separate frames, as shown in Figure 2 and subjected to computer processing. From the video selects the $n$-th and $(n+i)$-th frames in a row, where $n$ is the frame number and $i$ is a time interval, after which the matrix of pixels $(n+i)$-th frame are subtracted pixels of the $n$-th frame modulo for all three color channels.

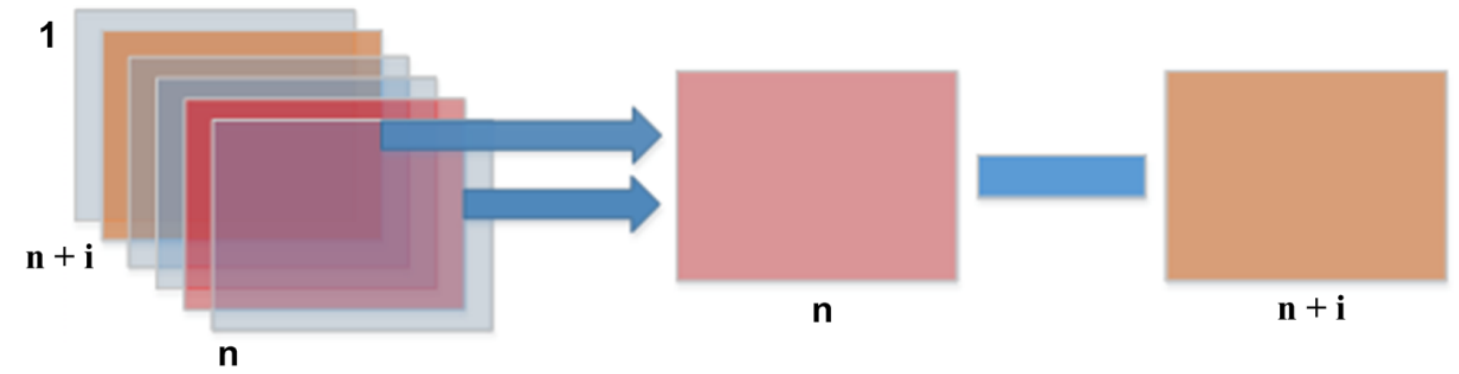

Figure 2: Sample of speckle image in the calculation of the intensities distributions difference. 
When processing is formed $R$ is the resulting matrix using the following data: $N_{1}$ the first frame of the selected pair; $N_{2}$ - the second frame of the selected pair; $N_{1}(x, y)_{r}$ - the intensity value of the red channel of the pixel in the $x$-th column of $y$-th row of the matrix of the first frame; according to equations:

$$
\begin{aligned}
& R(x, y)_{r}=N_{1}(x, y)_{r}+N_{2}(x, y)_{r}-2 * \min \left(N_{1}(x, y)_{r}, N_{2}(x, y)_{r}\right), \\
& R(x, y)_{g}=N_{1}(x, y)_{g}+N_{2}(x, y)_{g}-2 * \min \left(N_{1}(x, y)_{g}, N_{2}(x, y)_{g}\right), \\
& R(x, y)_{b}=N_{1}(x, y)_{b}+N_{2}(x, y)_{b}-2 * \min \left(N_{1}(x, y)_{b}, N_{2}(x, y)_{b}\right) .
\end{aligned}
$$

The resulting matrix $R$ is subjected to autocorrelation processing, results in a autocorrelation function (4) graph and the value of the speckle image energy spectrum (5). The last is obtained by applying discrete Fourier transform to the graph values and the summation of the squares of the obtained values is taken modulo.

TABLE 1: Results.

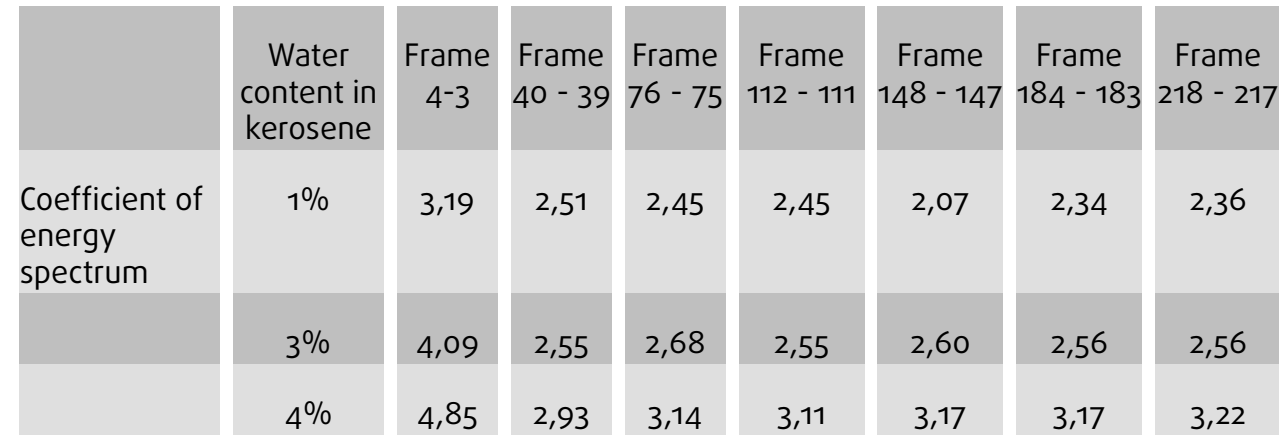

The more similar to each other two of the selected frame, the smaller the intensity and the lower is the ratio of the energy spectrum image.

$$
r_{\tau}=\frac{(n-\tau) \sum_{t=1}^{n-\tau} x_{t} x_{t+\tau}-\sum_{t=1}^{n-\tau} x_{t} \sum_{t=1}^{n-\tau} x_{t+\tau}}{\sqrt{(n-\tau) \sum_{t=1}^{n-\tau} x_{t}^{2}-\left(\sum_{t=1}^{n-\tau} x_{t}\right)^{2}} \sqrt{(n-\tau) \sum_{t=1}^{n-\tau} x_{t+\tau}^{2}-\left(\sum_{t=1}^{n-\tau} x_{t+\tau}\right)^{2}}}
$$

where $x_{t}$ - the brightness value of the pixel at the point $t ; x_{t+\tau}$ - the brightness value of the pixel at the point $\mathrm{t}$, at the time of displacement $\tau$; $\mathrm{n}$ - the number of pixels; $\tau-$ - step offset.

When calculating the coefficients of the energy spectrum function was used inverse discrete Fourier transform:

$$
x_{n}=\frac{1}{N} \sum_{k=1}^{K} X_{k} e^{\frac{-2 \pi i k n}{K}},
$$

where $N$ - the number of correlation function values, $K$ - the correlation function range. 

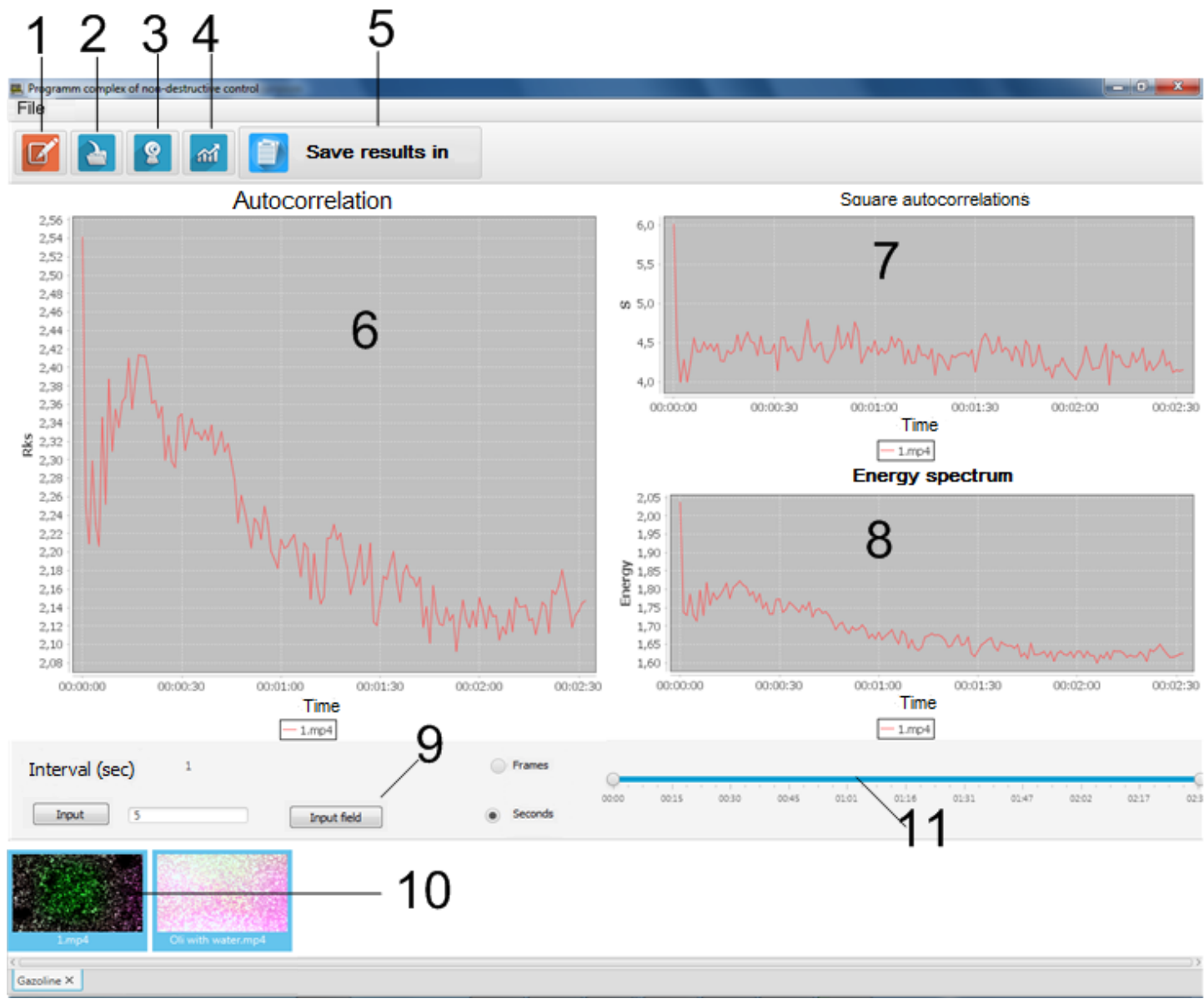

Figure 3: Interface of software module for the impurities determination in the working fluids composition for the speckle images parameters analysis. 1 - create of the new project; 2 - choice of speckle videos; 3 - device photo and video taking activation; 4 - calculation start; 5 - calculation results saving; 6 - the autocorrelation function graph; 7 - the square of autocorrelation graph; 8 - the energy spectrum graph; 9 - choice of the research realm video; 10 - field display the loaded video files; 11 - type of video processing; 12 - the interval recording selection.

\section{Software realization of a speckle images processing algorithms}

To the process automate of the impurities in working fluids determining of optical control method as a processing speckle photographs tool with the subsequent decision on the computed parameters, was developed a special software module. As programming language Java SE was used to determine the speckle images energy spectrum was applied the following free libraries: JTransforms to implement a fast discrete Fourier transform, and JFreeChart (AFreeChart) for plotting various types. The interface of this software is presented in Figure 3. This module allows to manage devices photo and video recording the process of sensing controlled liquid speckle-field, with the implementation of further phased array processing of the speckle images with the subsequent issuance to the operator of the results measurements parameters at a 


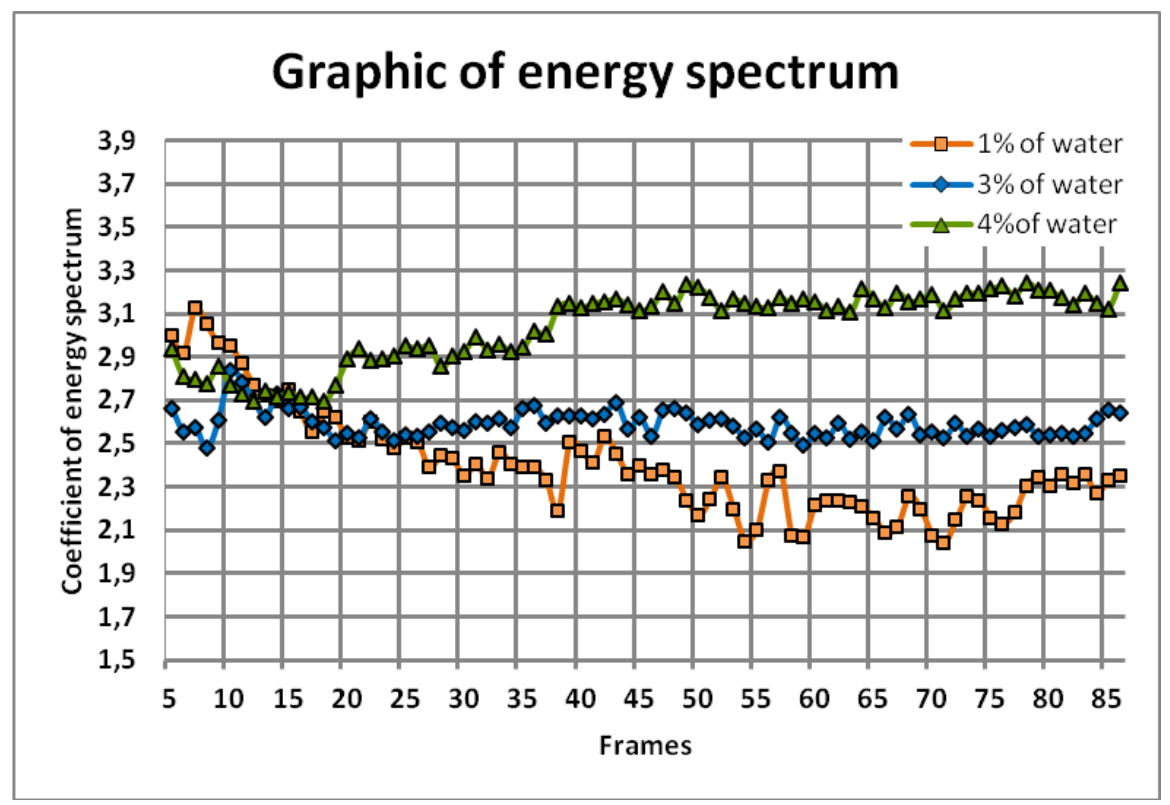

Figure 4: Depending on the ratio of the speckle-image energy spectrum from the frame number and the water content in kerosene percentage.

predetermined time interval from a perturbed state to a rest state (energy spectrum and the square of the speckle image autocorrelation function).

\section{A aviation fuel technical condition control}

For experimental studies were prepared 3 different samples of aviation fuel (TS-1) in the amount of $15 \mathrm{ml}$ each, with $1 \%, 3 \%$, and $4 \%$ content in its composition of water. Each sample was probed for 3 minutes, the result was 360 speckle image. Each array speckle pictures obtained from samples with different content of water was subjected to treatment, as a result of which a software module built diagram of dependence of the frame number (speckle image) from the value of its ratio in the energy spectrum. Obtained in the experiment course a results are presented in table 1 and Figure 4.

Thus, the graph shows changes of the energy spectrum as the attenuation of the impurities displacements in the cuvette with the investigated liquid, caused by forced oscillations (mixings). Graphs of the attenuation the energy spectrum coefficient allow to distinguish between cases with different amount of water in the kerosene.

\section{Engine oil control}

As a engine oil sample selected synthetic engine oil Toyota $5 \mathrm{~W}-40$. As reference was made by the sample of oil taken before operation and the remaining samples were 
prepared samples of pure oil with the addition of waste oil in the proportions: $1 \%$, $2 \%, 3 \%$ and $5 \%$ of its content, drained from the Toyota $3 \mathrm{MZ}$ engine of Toyota Harrier with total mileage $215000 \mathrm{~km}$ run after $10,000 \mathrm{~km}$. For researching was used the same setting as for the kerosene diagnostics. In the experiment, each sample was subjected to sensing, the result is recorded speckle image are presented in table 2 (top row). Further, the speckle image was subjected to autocorrelation function, which was determined by energy spectrum $\mathrm{F}_{k}$, shown in Figure 5 and its width - the lower row of table 2.

TABLE 2: Results of the experiment for impurities determination in the engine oil composition.

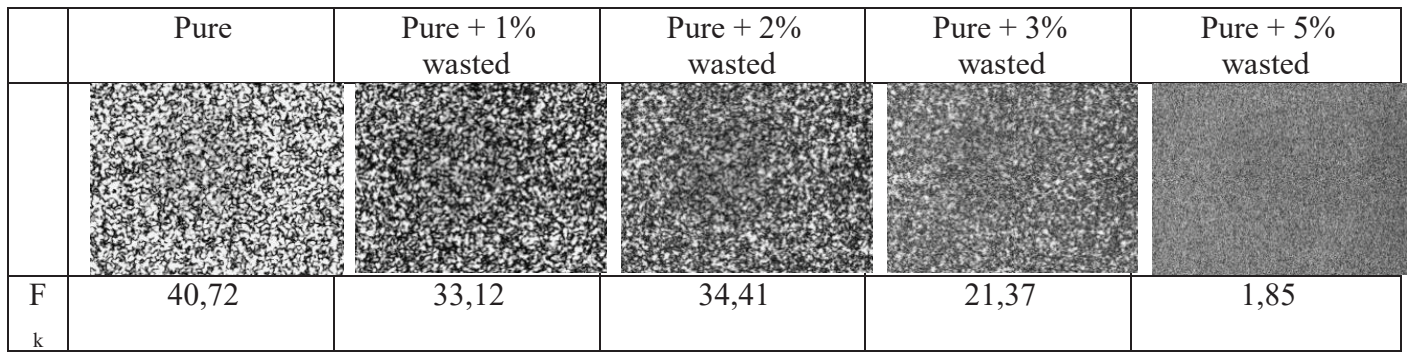

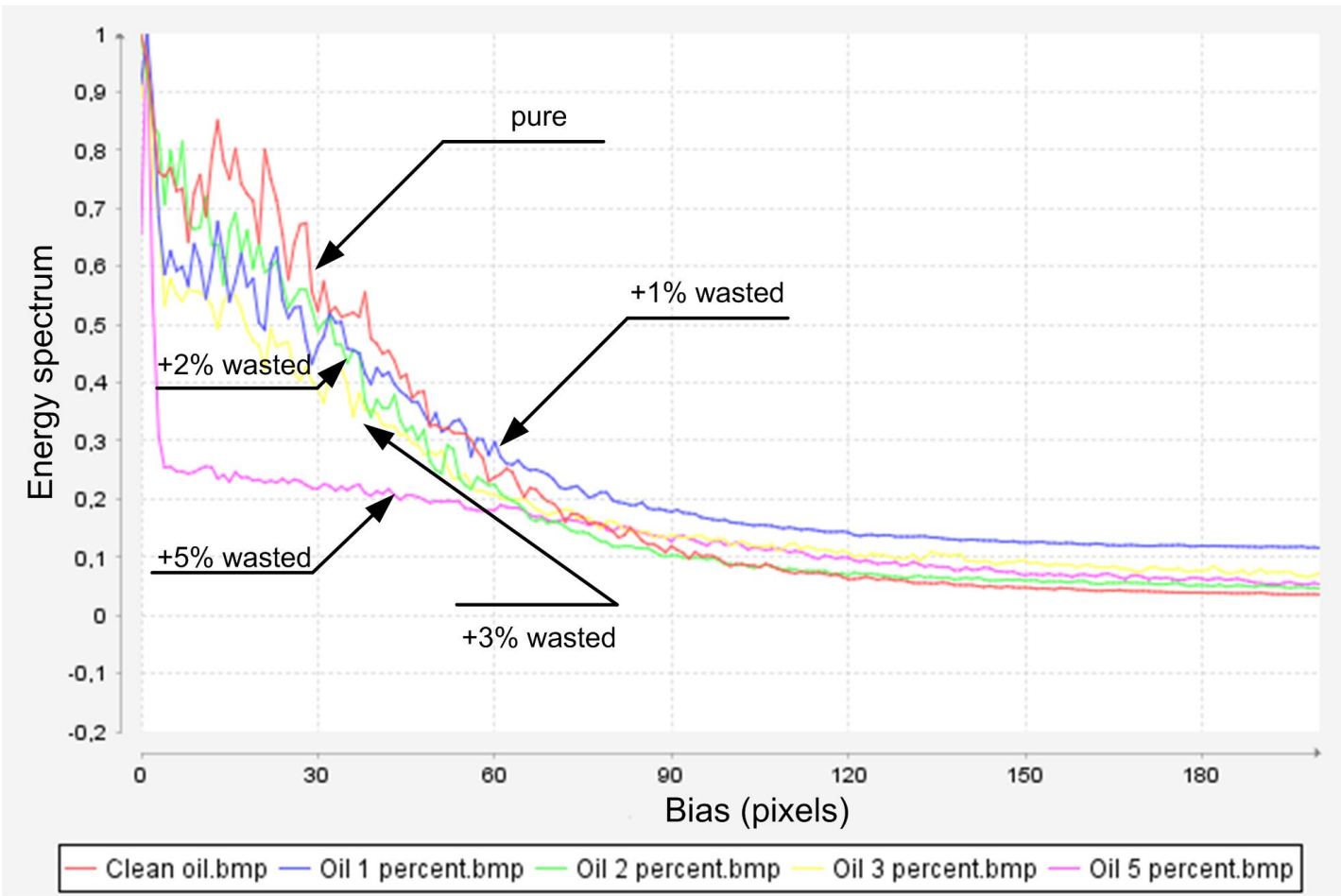

Figure 5: Graphs of the speckle patterns energy spectrum from oil samples with different waste oil percentages. 


\section{Conclusion}

Thus, as a research result, the method application of operating fluids diagnosis, it is established that the proposed speckle photography processing algorithms used in specialized software and the installation, allow for the assessment of technical condition of aviation fuel and motor oil. The application of the chosen method for the fluids control requires further study and to develop procedures for the specific working fluid control.

\section{References}

[1] Suslin M.A., Prishchepenko V. V., Melkumov V. N. Study of losses in the fuels with dissolved and emulsified moisture at microwave frequencies // Izmeritelnaya technical, 2016. №3. pp.68-71.

[2] Kazmin A.I. Microwave method and device of the analytical express control the quality of aviation fuel. Electronic journal «Trudi MAl». Vipusk №74: http://trudymai.ru/published.php?ID=49143.

[3] Tuchin V.V. Optical biomedical diagnostics. Moscow: Izdatelstvo Fizikomatematicheskoy literaturi, 2007, T.1560 p.

[4] A. P. Vladimirov, A. A. Baharev, A. S. Malygin, J. A. Mikhailova, I. A. Novoselova, D. I. Yakin. Application of speckle dynamics for studies of cell metabolism // Proc. of SPIE, 2015, v. 9529, 95291F-1 - 95291F-10.

[5] Fedoretz A.A., Bezugliy B.A. Express-method of identification and quality control of liquid. Patent RF №2247968, 10.03.2005.

[6] Medvedeva S.Y. Creating a laser photometer for the analysis of trace impurities in solutions and gases // Sbornik nauchnih trudov «Nauchnaya sessia NIYU MIFI-2011» PP.59-60.

[7] Vovk T.A., Petrov N.V. Correlation characterization of particles in volume based on peak-to-basement ratio // Scientific reports. 2017. T.7. 